\title{
A linear model based noise evaluation of a capacitive servo-accelerometer fabricated by MEMS
}

\author{
Yusaku Yoshida ${ }^{1,2}$, Hideaki Kakuma ${ }^{1}$, Hiroshi Asanuma ${ }^{2}$, \\ and Hiroaki Niitsuma ${ }^{2}$ \\ ${ }^{1}$ Device Technology Development Department, \\ Kawasaki Research and Development Center, Mitutoyo Corporation \\ 1-20-1 Sakado Takatsu-ku Kawasaki, 213-8533, Japan \\ ${ }^{2}$ Graduate School of Environmental Studies, Tohoku University \\ 6-6-20 Aramaki Aza Aoba Aoba-ku, Sendai, 980-8579, Japan
}

\begin{abstract}
The noise characterization of a capacitive servo-accelerometer using a linear model is presented in this paper. The input reference noise of electronic circuit is expressed as a capacitive variation and it is introduced to a linear model for frequency response, separately with the thermal mechanical noise. The simulated noise was generally found to conform to the measured noise in the experiment, suggesting that the model can be effectively used for noise evaluation of accelerometers fabricated in a similar fashion.
\end{abstract}

Keywords: MEMS accelerometer, noise, linear model

Classification: Micro- or nano-electromechanical systems

\section{References}

[1] H. Niitsuma, "Concept of Subsurface Micro-sensing," Proc. 96th SEGJ Conf., pp. 33-34, 1997. (in Japanese)

[2] T. B. Gabrielson, "Mechanical-Thermal Noise in Micromachined Acoustic and Vibration Sensors," IEEE Trans. Electron Devices, vol. ED-40, pp. 903-909, 1993.

[3] R. P. van Kampen, Bulk-Micromachined Capacitive Servo-Accelerometer, Delft University Press, 1995.

[4] S. Fukui and R. Kaneko, "Analysis of ultra-thin gas film lubrication based on linearized Boltzman equation: first report-derivation of a generalized lubrication equation including thermal creep flow," J. Tribol. Trans. AMSE, vol. 110, pp. 253-262, April 1988.

[5] T. Veijola, H. Kuisma, J. Lahdenpera, and T. Ryhanen, "Equivalentcircuit model of the squeezed gas film in a silicon accelerometer," Sensors and Actuators, A48, pp. 239-248, Oct. 1995. 


\section{Introduction}

The authors have been developing a capacitive servo-accelerometer by using the MEMS technologies under the "Sub-surface Microsensing Project" in Tohoku University since 1993 [1]. In its current state the servo-accelerometer has achieved a frequency range from $\mathrm{DC}$ to $250 \mathrm{~Hz}$ and an input reference noise of $10 \mu \mathrm{G} / \sqrt{\mathrm{Hz}}$. A linear model was developed to analyze the frequency response of the accelerometer. For practical purposes, it became apparent that a noise model would also be required for noise simulation and evaluation.

The accelerometer includes the thermal mechanical noise [2], caused by Brownian motion of air, and the electronic noise. Kampen [3] proposed a theoretical noise model of a capacitive servo-accelerometer including the thermal mechanical noise and the electronic noise. Although not verified through experiments, it was predicted by Kampen that at a low frequency range the mechanical noise is dominant and at a high frequency range the electronic noise becomes dominant as the forward gain increases.

In Kampen's theoretical noise model, the input reference noise of an electronic circuit is expressed as the displacement of the mass, which requires electronic and mechanical characteristics to estimate the value. However the electronic noise is inherently different from the mechanical noise, so that the input reference noise of the circuit should not include the mechanical characteristics.

In this paper, the input reference noise is expressed as capacitance variation, which does not include mechanical characteristics, and it is introduced to a linear model developed for frequency response, separately with the thermal mechanical noise. The model was verified by comparing the simulated noise spectrum with measured data, and in general the agreement was quite good although there are some discrepancies.

\section{A linear model for accelerometer noise}

Fig. 1 a shows a simple schematic of a mechanical part of a capacitive accelerometer which consists of springs, mass, air dumper and fixed electrodes. When the acceleration is applied to the accelerometer, the mass moves and capacitance between the fixed electrode and the surface of the mass varies. The circuit in Fig. $1 \mathrm{~b}$ detects the capacitance variation and converts it to the voltage.

Two noise sources were considered in the accelerometer; one is the thermal mechanical noise and the other is the electronic circuit noise.

The thermal mechanical noise $F_{n}[2]$, caused by Brownian motion of air, works as a force acting on the mass and is described by

$$
F_{n}=\sqrt{4 \lambda_{a} k_{b} T}
$$

where $\lambda_{a}$ is the damping coefficient, $k_{b}$ is Boltzman's constant and $T$ is temperature. $\lambda_{a}$ can be calculated from the generalized lubrication equation which describes the squeezed film effect $[4,5]$. 
The circuit noise is usually referred as the input reference noise. The input reference noise of the circuit may be expressed in many ways, such as voltage, current, displacement of the mass, and so on. However the input of the circuit is capacitance variation, so that it should be employed as the input reference noise of the electronic circuit. The capacitance variation can

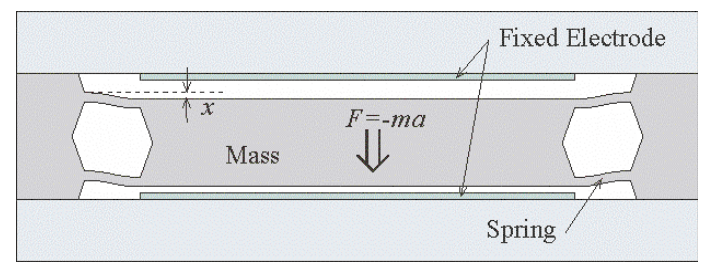

(a)

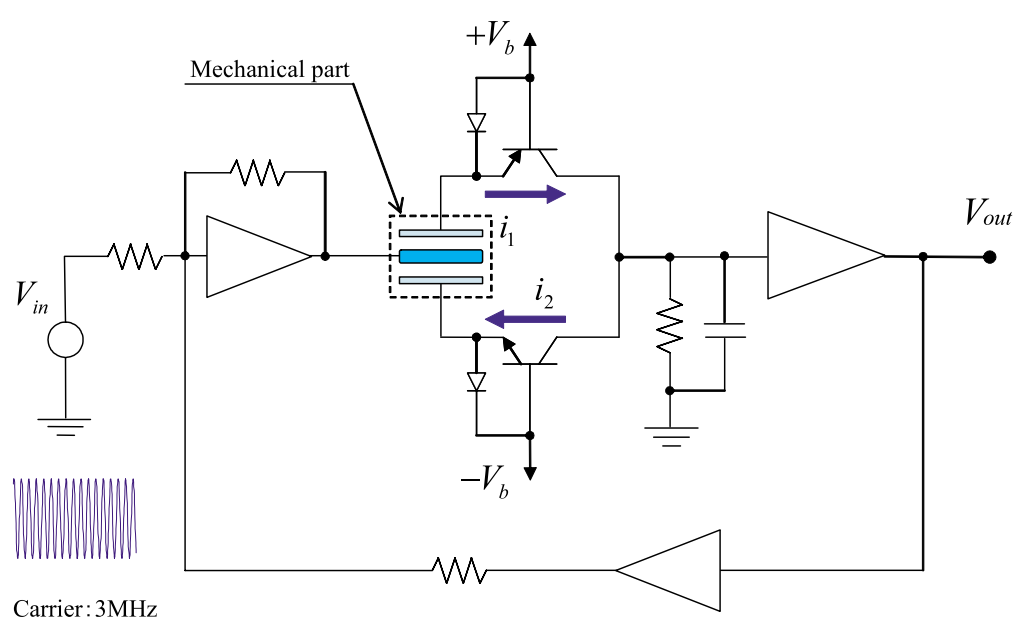

(b)

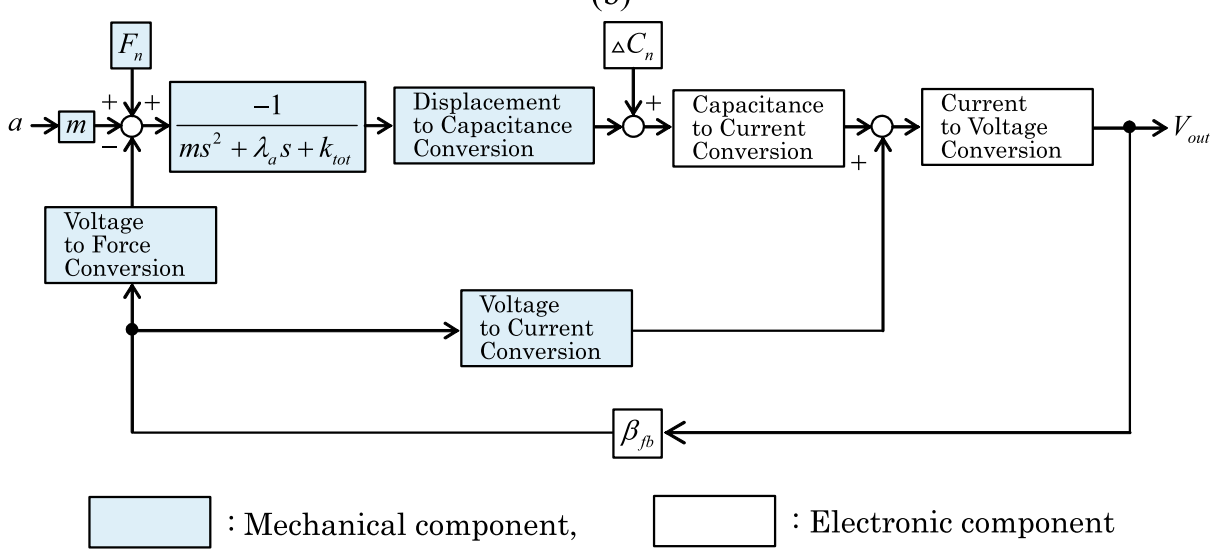

(c)

Fig. 1. Outline of the capacitive servo-accelerometer and the block diagram

(a) Mechanical model of the capacitive accelerometer

(b) Electronic circuit of the capacitive servoaccelerometer

(c) A linear model for frequency response, with the thermal mechanical noise and the input reference noise of an electronic circuit for noise characterization. 
be evaluated using only the circuit characteristics without the requirement of mechanical characteristics.

Fig. $1 \mathrm{c}$ shows the linear model developed for frequency response including the configuration of the noise sources. The thermal mechanical noise $F_{n}$ is placed at the node which represents the force acting on the mass. The input reference noise of the circuit $\Delta C_{n}$ is introduced at the node that connects the mechanical part with the circuit.

To utilize this model, the thermal mechanical noise $F_{n}$ and input reference noise of electronic circuit $\Delta C_{n}$ must be calculated beforehand. In this paper, the thermal mechanical noise $F_{n}$ is calculated from the Eq. (1) and the input reference noise of the electronic circuit $\Delta C_{n}$ is calculated from the experiment using the actual circuit.

\section{Input reference noise of the electronic circuit}

The input reference noise is calculated using the following formula,

$$
\Delta C_{n}=\frac{V_{R_{1}}}{K_{C-V}}
$$

where $V_{R_{1}}$ is the output noise and $K_{C-V}$ is the C-V conversion gain. Hence, to calculate the input reference noise $\Delta C_{n}, V_{R_{1}}$ and $K_{C-V}$ must first be evaluated.

To evaluate the output noise, we prepared an actual circuit. Fig. 2 shows the setup of the experiment and the output noise measured. The circuit was set up with an open loop configuration, where two ceramic condensers are connected instead of a mechanical part. These ceramic condensers enable us to prevent the occurrence of thermal mechanical noise, by not allowing capacitance fluctuation. Under the same conditions of the feedback system, $V_{b}=6 \mathrm{~V}$ were applied to the condensers and the carrier frequency was adjusted to $3 \mathrm{MHz}$. Also complying with the feedback system, the first order low-pass filter at $723 \mathrm{~Hz}$ and the second order at $1 \mathrm{kHz}$ were added to the circuit. To measure the output noise, the FFT signal analyzer HP35685A was used. The output noise was found to be $2 \mu \mathrm{V} / \sqrt{\mathrm{Hz}}$.

To evaluate the $\mathrm{C}-\mathrm{V}$ conversion gain, the circuit simulator PSpice was used employing the same circuit configuration as in the experiment to evaluate the output noise. The value of the $\mathrm{C}-\mathrm{V}$ conversion gain was found to be $5.19 \times 10^{12} \mathrm{~V} / \mathrm{F}$.

Substituting the above values into Eq. (2) produced the result $0.4 \times$ $10^{-18} \mathrm{~F} / \sqrt{\mathrm{Hz}}$ as the input reference noise. This value is also indicated in Fig. 2.

The $1 / f$ noise occurring at less than $5 \mathrm{~Hz}$ was assumed to be a by-product of the measurement setup and therefore was not included in the linear model.

\section{Evaluation of the linear model and noise characteristics}

To evaluate the linear model described above, we compare an accelerometer's simulated noise spectrum with the measured noise spectrum of a fabricated capacitive servo-accelerometer. 


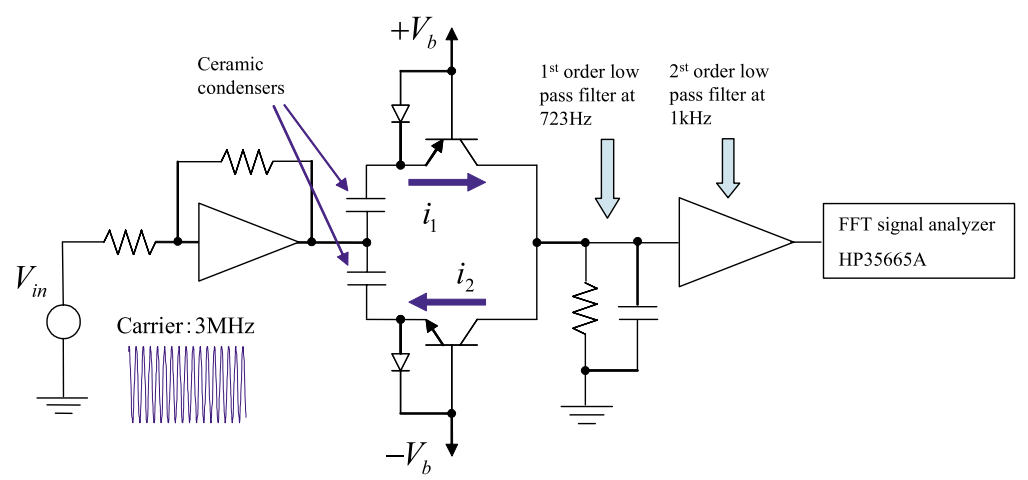

(a)

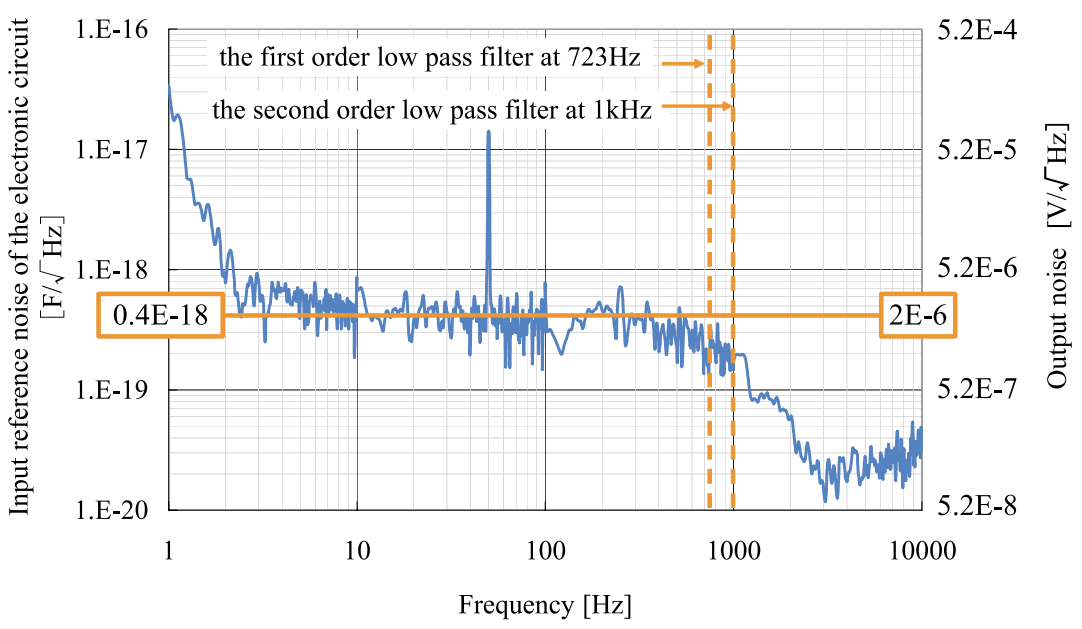

(b)

Fig. 2. The electronic circuit; the output noise and the input reference noise.

(a) The electronic circuit and the setup for the noise measurement.

(b) The output noise and the input reference noise.

A fabricated accelerometer was set up for its noise measurement by adjusting the frequency response from DC to $150 \mathrm{~Hz}$ to within $10 \%$ without resonance and the sensitivity to $1 \mathrm{~V} / \mathrm{G}$. The measured noise is shown as the input reference noise of the accelerometer in Fig. 3. From 0.3 to $50 \mathrm{~Hz}$, it shows the noise spectrum is flat. At the frequency above $50 \mathrm{~Hz}$ the noise increases and around $400 \mathrm{~Hz}$ it starts decreasing. At the frequency below $0.3 \mathrm{~Hz}, 1 / f$ noise seems to occur.

To simulate the noise of accelerometer, $0.63 \times 10^{-9} \mathrm{~N} / \sqrt{\mathrm{Hz}}$ was obtained from the Eq. (1) for the thermal mechanical noise and the input reference noise was estimated as $0.4 \times 10^{-18} \mathrm{~F} / \sqrt{\mathrm{Hz}}$ through the experiment described before. The simulated result using these values is indicated in Fig. 3 along with the measured noise.

It was found that depending on the frequency range, the noise sources' dominance changes. The thermal mechanical noise surpasses the circuit noise at below $100 \mathrm{~Hz}$, whereas at above $100 \mathrm{~Hz}$ the circuit noise is dominant. This 


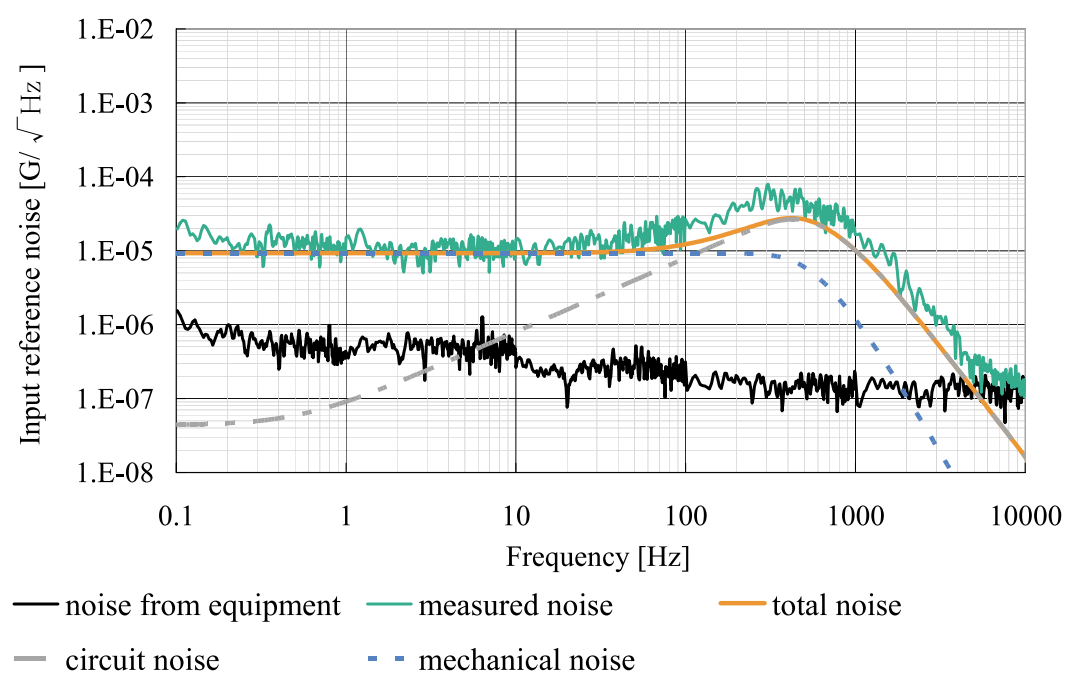

Fig. 3. Noise comparison between simulated and measured result.

result confirms Kampen's prediction [3] regarding the behavior of noise as the frequency changes.

When comparing the simulated and measured noise, there is a discrepancy over the frequency range where the electronic noise is dominant. This may have been caused by estimating the circuit noise lower than the real value. However the simulated noise spectrum is generally shown to match the measured noise spectrum. Overall, the linear model proves to be highly useful in clarifying noise characteristics of the capacitive servo-accelerometer.

\section{Conclusions}

A linear model for noise characterization of a capacitive servo-accelerometer was realized by introducing the thermal mechanical noise and the input reference noise of an electronic circuit to a model for frequency response. The input reference noise of accelerometer was calculated through the theoretical estimation of the thermal mechanical noise and the experimental estimation of the input reference noise of the electronic circuit. The simulated result was found to generally match the measured noise. This result suggests that the model can be highly practical and effective in evaluating noise characteristics.

One of the main advantages of this model is that the input reference noise of an electronic circuit is employed as the capacitance variation evaluated purely from the circuit noise, thus doing away with the necessity for mechanical characteristics as required in Kampen's theoretical noise model.

\section{Acknowledgments}

This work presented in this paper is supported by the following funds; Japan society for the Promotion of Science, and New Energy and Industrial Technology Development Organization, Japan (Reserch Matching Funds Approach, No.31). 
A special word of thanks to Kazusaku Tezuka, President of Mitutoyo Corporation, Katsuhisa Chikahata, former President of Akashi Corporation, and Sadayuki Matsumiya, Director of Mitutoyo Corporation. The authors also with to acknowledge Prof. M. Esashi, NiCHE Tohoku University for his commnents and encouragement to complete this paper. 